\title{
Top quark pair production in the exclusive processes at the LHC
}

\author{
Victor P. Gonçalves $\odot,{ }^{1, *}$ Daniel E. Martins, ${ }^{2, \dagger}$ Murilo S. Rangel ${ }^{2,+\star}$ and Marek Tasevsky $\oplus^{3, \S}$ \\ ${ }^{1}$ Instituto de Física e Matemática, Universidade Federal de Pelotas (UFPel), \\ Caixa Postal 354, CEP 96010-090 Pelotas, Rio Grande do Sol, Brazil \\ ${ }^{2}$ Instituto de Física, Universidade Federal do Rio de Janeiro (UFRJ), \\ Caixa Postal 68528, CEP 21941-972 Rio de Janeiro, Rio de Janeiro, Brazil \\ ${ }^{3}$ Institute of Physics of the Czech Academy of Sciences, Na Slovance 2, 18221 Prague 8, Czech Republic
}

(Received 10 July 2020; accepted 2 October 2020; published 21 October 2020)

\begin{abstract}
We analyze the LHC prospects for measurements of the $t \bar{t}$ pair produced exclusively in photon-photon or semiexclusively in photon-Pomeron and Pomeron-Pomeron processes using protons tagged in forward proton detectors on both sides of the interaction point. These processes are interesting from the point of view of a possible measurement of the top quark mass and constraining models used in beyond the Standard Model physics. Focusing on the semileptonic channel, $t \bar{t} \rightarrow j j b l \nu_{l} \bar{b}$, making use of the exclusive nature of the final state, together with the use of timing information provided by forward proton detectors, relevant exclusive and inclusive backgrounds are studied in detail for different luminosity (or pileup) scenarios and found to be important for further consideration. While good prospects are found for observing the signal, the top quark mass measurement turns out not to be competitive with measurements in inclusive channels.
\end{abstract}

DOI: 10.1103/PhysRevD.102.074014

\section{INTRODUCTION}

One of the more important processes for studying perturbative quantum chromodynamics (pQCD) is the heavy quark production in hadronic collisions (for a review, see, e.g., Ref [1]). Such a process is expected to improve the description of the measured data by $\mathrm{pQCD}$ at high energies, and it is also important background material for analyses searching for signals of beyond the Standard Model (BSM) processes. In other words, an analysis of the top quark production allows us to constrain input parameters for pQCD predictions and probe different BSM scenarios [2-4]. This expectation is directly related to the fact that the top quark couples to all gauge bosons and the Higgs boson, which implies that the top production is very sensitive to the presence of BSM phenomena. In addition, recent experimental results for the inclusive top pair production have demonstrated that this process can be used to measure the top mass in a well-defined scheme with high accuracy [5-10]. These results provide motivation for

\footnotetext{
*barros@ufpel.edu.br

†dan.ernani@gmail.com

*murilo.rangel@ufrj.br

\$Marek.Tasevsky@cern.ch
}

Published by the American Physical Society under the terms of the Creative Commons Attribution 4.0 International license. Further distribution of this work must maintain attribution to the author(s) and the published article's title, journal citation, and DOI. Funded by SCOAP. the study of the top pair production in diffractive processes, where the final state is cleaner than the typical inclusive one, where both incident protons fragment and a large number of particles are produced in addition to the top pair (for a review on diffraction see, e.g., Ref. [11]). In our analysis we will focus on the top pair production in photonand pomeron-induced processes at the center of system energy, $\sqrt{s}$, of $13 \mathrm{TeV}$, where both incident protons remain intact in the final state. In principle, such events can be collected using forward proton detectors (FPDs) such as the ATLAS Forward Proton detector (AFP) $[12,13]$ and CMS/ TOTEM Precision Proton Spectrometer [14] that are installed symmetrically around the interaction point at a distance of roughly $210 \mathrm{~m}$ from the interaction point. We will restrict our analysis to Standard Model subprocesses and postpone the study of the impact of new physics on the diffractive $t \bar{t}$ production to a future publication.

At high energies the top pair production in hadronic collisions is dominated by gluon-gluon interactions, provided that the incident hadrons break up. However, a top pair can also be generated in photon-photon [Fig. 1(a)], photonPomeron [Fig. 1(b)] and Pomeron-Pomeron [Fig. 1(c)] interactions. Since the photon and Pomeron are color-singlet objects, these processes are characterized by the presence of two regions devoid of hadronic activity, called rapidity gaps, separating the intact very forward protons from the central massive object. Moreover, the Fig. 1(a) process is a typical example of an exclusive process, where nothing is produced except the leading hadrons and the central object. In contrast, if we assume that the Pomeron has a partonic 


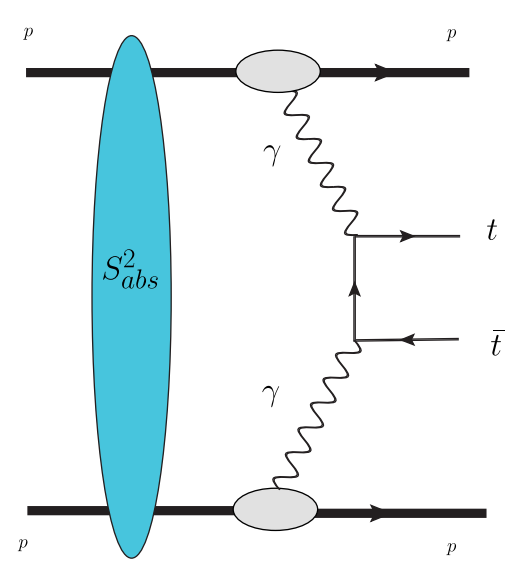

(a)

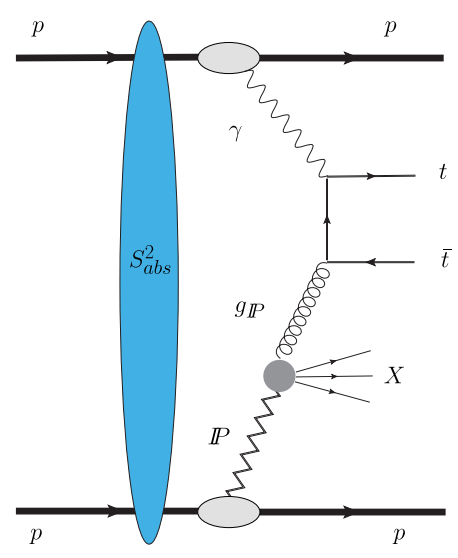

(b)

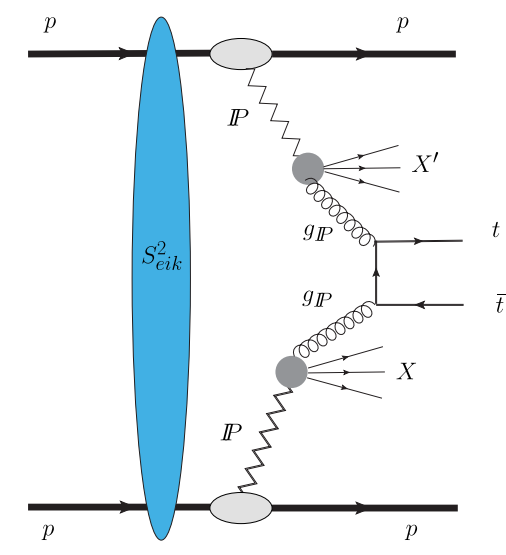

(c)

FIG. 1. Top pair production in (a) photon-photon, (b) photon-Pomeron, and (c) Pomeron-Pomeron interactions in $p p$ collisions.

structure [15], then in the Fig. 1(b) and Fig. 1(c) processes rapidity gaps can be filled by particles from fragmenting Pomeron remnants. The large invariant mass of the produced system implies that the intact protons in the final state can be tagged by FPDs. Consequently, such events can, in principle, be separated and used to improve our understanding of top quark production. Our goal in this paper is to perform a detailed analysis of top pair production considering the processes shown in Fig. 1 and to present expected event yields that take into account the current detector acceptances and pileup effects expected for the next run of the LHC. In order to obtain realistic predictions for the top pair production in photon- and Pomeron-induced interactions and to be able to include experimental cuts in the calculations, the treatment of these processes in a Monte Carlo simulation is indispensable. Some years ago, Forward Physics Monte Carlo (FPMC) [16] was generalized in order to simulate the central particle production with one or two leading protons and some hard scale in the event. In this paper we use this generator to estimate the top pair production at the LHC. We try to give realistic estimates of the event yields for the signal as well as the backgrounds, and we also comment on the possibility of extracting information about the top quark mass using FPDs.

This paper is organized as follows. In Sec. II we present a brief review of the formalism for the top pair production in photon- and Pomeron-induced interactions in $p p$ collisions. In Sec. III we discuss details of the selection of events and cuts implemented in our analysis, concentrating on collisions at $\sqrt{s}=13 \mathrm{TeV}$. In Sec. IV we present our predictions for the invariant mass and transverse momentum distributions as well as for the total cross sections for the top pair production in $\gamma \gamma, \gamma \mathbb{P}$, and $\mathbb{P P}$ nteractions. Finally, in Sec. V we summarize our main conclusions.

\section{FORMALISM}

An ultrarelativistic charged hadron (proton or nucleus) gives rise to strong electromagnetic fields, such that the photon stemming from the electromagnetic field of one of the two colliding hadrons can interact with one photon of the other hadron (photon-photon process) or can interact directly with the other hadron (photon-hadron process) $[17,18]$. The total cross section for the photon-induced interactions can be factorized in terms of the equivalent flux of photons into the hadron projectiles and the photon-photon or photon-target production cross section. In particular, the top pair production in photon-photon interactions represented in Fig. 1(a) is described by

$$
\begin{aligned}
& \sigma\left(h_{1} h_{2} \rightarrow h_{1} \otimes t \bar{t} \otimes h_{2}\right) \\
& \quad=\int d x_{1} \int d x_{2} \gamma_{1}\left(x_{1}\right) \cdot \gamma_{2}\left(x_{2}\right) \cdot \hat{\sigma}(\gamma \gamma \rightarrow t \bar{t}),
\end{aligned}
$$

where $\otimes$ represents the presence of a rapidity gap in the final state, $x$ is the fraction of the hadron energy carried by the photon, and $\gamma(x)$ is the equivalent photon distribution of the hadron. The general expression for the photon flux of the proton is given by [19]

$$
\begin{aligned}
\gamma(x)= & -\frac{\alpha}{2 \pi} \int_{-\infty}^{-\frac{m^{2} x^{2}}{1-x}} \frac{d t}{t}\left\{\left[2\left(\frac{1}{x}-1\right)+\frac{2 m^{2} x}{t}\right] H_{1}(t)\right. \\
& \left.+x G_{M}^{2}(t)\right\},
\end{aligned}
$$

where $t=q^{2}$ is the momentum transfer squared of the photon,

$$
H_{1}(t) \equiv \frac{G_{E}^{2}(t)+\tau G_{M}^{2}(t)}{1+\tau},
$$

with $\tau \equiv-t / m^{2}$ and $m$ being the nucleon mass, and where $G_{E}$ and $G_{M}$ are the Sachs elastic form factors. In what follows we will use the photon flux derived in Ref. [18], where an analytical expression is presented 
(for a recent study of the $t \bar{t}$ production in $\gamma \gamma$ interactions see, e.g., Ref. [20]).

The photon-hadron processes can be classified as inclusive or diffractive, depending upon whether the proton breaks up or remains intact, respectively. The inclusive $t \bar{t}$ production by inclusive $\gamma p$ interactions in $p p$ collisions at the LHC was analyzed in Ref. [21]. In our study we are interested in diffractive photon-hadron case, with the diffractive interaction being described by a Pomeron exchange. The cross section for the top pair production in a photon-Pomeron interaction, represented in Fig. 1(b), is given by

$$
\begin{aligned}
& \sigma\left(h_{1} h_{2} \rightarrow h_{1} \otimes t \bar{t} X \otimes h_{2}\right) \\
& =\int d x_{1} \int d x_{2}\left[g_{1}^{D}\left(x_{1}, \mu^{2}\right) \cdot \gamma_{2}\left(x_{2}\right)\right. \\
& \left.\quad+\gamma_{1}\left(x_{1}\right) \cdot g_{2}^{D}\left(x_{2}, \mu^{2}\right)\right] \cdot \hat{\sigma}(\gamma g \rightarrow t \bar{t}),
\end{aligned}
$$

where $g^{D}\left(x, \mu^{2}\right)$ is the diffractive gluon distribution, whose evolution is described by the Dokshitzer-Gribov-LipatovAltarelli-Parisi (DGLAP) evolution equations and is determined from events with a rapidity gap or intact proton, mainly at HERA [11]. Similarly, the top pair can be produced by Pomeron-Pomeron interactions as represented in Fig. 1(c), with the cross section given by

$$
\begin{aligned}
& \sigma\left(h_{1} h_{2} \rightarrow h_{1} \otimes X t \bar{t} X^{\prime} \otimes h_{2}\right) \\
& \quad=\int d x_{1} \int d x_{2} g_{1}^{D}\left(x_{1}, \mu^{2}\right) \cdot g_{2}^{D}\left(x_{2}, \mu^{2}\right) \cdot \hat{\sigma}(g g \rightarrow t \bar{t}) .
\end{aligned}
$$

In the resolved Pomeron model [15], the diffractive gluon distribution in the proton, $g^{D}\left(x, \mu^{2}\right)$, is defined as a convolution of the Pomeron flux emitted by the proton, $f_{\mathbb{P}}\left(x_{\mathbb{P}}\right)$, and the gluon distribution in the Pomeron, $g_{\mathbb{P}}\left(\beta, \mu^{2}\right)$, where $\beta$ is the momentum fraction carried by the struck parton inside the Pomeron. The Pomeron flux is given by $f_{\mathbb{P}}\left(x_{\mathbb{P}}\right)=\int_{t_{\min }}^{t_{\max }} d t f_{\mathbb{P} / \mathfrak{p}}\left(x_{\mathbb{P}}, t\right)$, where $f_{\mathbb{P} / \mathfrak{p}}\left(x_{\mathbb{P}}, t\right)=$ $A_{\mathbb{P}} \cdot \frac{e^{B_{\mathbb{P}} t}}{x_{\mathbb{P}}^{2 \mathbb{P}_{\mathbb{P}}(t)-1}}$ and $t_{\min }, t_{\max }$ are kinematic boundaries. The Pomeron flux factor is inspired by Regge theory, where the Pomeron trajectory is assumed to be linear, $\alpha_{\mathbb{P}}(t)=$ $\alpha_{\mathbb{P}}(0)+\alpha_{\mathbb{P}}^{\prime} t$, and the parameters $B_{\mathbb{P}}, \alpha_{\mathbb{P}}^{\prime}$ and their uncertainties are obtained from fits to $\mathrm{H} 1$ data [22]. The diffractive gluon distribution is then given by

$$
\begin{aligned}
g^{D}\left(x, \mu^{2}\right) & =\int d x_{\mathbb{P}} d \beta \delta\left(x-x_{\mathbb{P}} \beta\right) f_{\mathbb{P}}\left(x_{\mathbb{P}}\right) g_{\mathbb{P}}\left(\beta, \mu^{2}\right) \\
& =\int_{x}^{1} \frac{d x_{\mathbb{P}}}{x_{\mathbb{P}}} f_{\mathbb{P}}\left(x_{\mathbb{P}}\right) g_{\mathbb{P}}\left(\frac{x}{x_{\mathbb{P}}}, \mu^{2}\right) .
\end{aligned}
$$

A similar definition can be established for the diffractive quark distributions. In our analysis we will include the quark contributions for the top pair production, associated, e.g., with the $q \bar{q} \rightarrow t \bar{t}$ subprocess, and the diffractive parton distribution will be described by the parametrization obtained by the H1 Collaboration at DESY-HERA, denoted as fit A in Ref. [22]. For a similar analysis of charm and bottom production in $\gamma \mathbb{P}$ and $\mathbb{P P}$ interactions see, e.g., Refs. [23-25]. Our predictions for the $t \bar{t}$ production could potentially be sensitive to the Pomeron gluon distribution at large values of $\beta$ and $\mu^{2}$, beyond the kinematical range probed by HERA. The behavior of $g_{\mathbb{P}}$ in this region is driven by the DGLAP evolution equations. As the inclusive $t \bar{t}$ data are quite well described by predictions derived using these equations (see, e.g., Ref. [7]) and the results presented in Ref. [26] indicate that the uncertainty on $g_{\mathbb{P}}$ is small in that kinematical range, we expect, as well, a small impact on our predictions associated with the choice of the diffractive parton distribution.

One important open question is the treatment of additional soft interactions between incident protons, which leads to an extra production of particles that destroys the rapidity gaps in the final state [27]. As these effects have a nonperturbative nature, they are difficult to treat and their magnitude is strongly model dependent (for reviews see Refs. [28-30]). For photon-photon and photon-Pomeron interactions, the contribution of the soft interactions, represented by the factor $S_{a b s}^{2}$ in Figs. 1(a) and 1(b), is expected to be small due to the long duration of the electromagnetic interaction. As a consequence, in what follows we will assume that $S_{a b s}^{2}=1$. In contrast, for Pomeron-Pomeron interactions, the impact of soft interactions is non-negligible, implying the violation of the QCD hard scattering factorization theorem for diffraction in $p p$ collisions [31]. Assuming that the hard process occurs on a short enough timescale that the physics that generate the additional particles can be factorized, the inclusion of these additional absorption effects can be parametrized in terms of an average rapidity gap survival probability, $S_{\text {eik }}^{2}$. Such a quantity corresponds to the probability of the scattered proton not dissociating due to the secondary interactions. The gap survival probability has been calculated considering different approaches, giving distinct predictions (see, e.g., Ref. [32]). As in previous studies for single- and double-diffractive production [24,25,33-35] we also follow this simplified approach assuming that $S_{e i k}^{2}=0.03$ for Pomeron-Pomeron interactions, as predicted in Ref. [36]. However, it is important to emphasize that the magnitude of the rapidity gap survival probability is still an open question.

\section{EXPERIMENTAL PROCEDURE}

In this section, we explain the cuts used to discriminate the signal from the background sources while considering the pileup effect. We provide results for four working points regarding the instantaneous luminosity (and hence the average amount of pileup interaction per bunch crossing, $\langle\mu\rangle$ ) and assume integrated luminosity in each of the 
working points. We consider these values of $\langle\mu\rangle$ and the corresponding integrated luminosity: $\langle\mu\rangle=0 \quad\left(1 \mathrm{fb}^{-1}\right)$, $5\left(10 \mathrm{fb}^{-1}\right), 10\left(30 \mathrm{fb}^{-1}\right)$, and $50\left(300 \mathrm{fb}^{-1}\right)$. The separation of the (semi)exclusive $t \bar{t}$ signal from backgrounds at $13 \mathrm{TeV}$ collisions proceeds in two steps: first we select the central system as in the inclusive processes, then we apply exclusivity criteria. We select the so-called semileptonic $t \bar{t}$ decays: $t \bar{t} \rightarrow j j b l \nu_{l} \bar{b}$, (one top quark decaying hadronically into two light quarks and a $b$-quark, the other into a $b$ quark and a $W$ boson, which then decays leptonically into a lepton and neutrino). In inclusive interactions, the semileptonic channel was shown to give an optimum signal yield while keeping the purity of the signal still reasonably high. For example, for the ATLAS semileptonic channel at $8 \mathrm{TeV}$ [37] the total background contamination was kept at a level of $10 \%$, with single-top and $W+$ jets background processes contributing most, both by about $3.5 \%-4 \%$, with the rest coming from $Z+$ jets and multijet backgrounds. Thus, by using the same cuts as in [37], we ensure that the backgrounds from the four inclusive processes above are kept reasonably low even in the presence of pileup. In the second step, we make use of the (semi)exclusive nature of our signal and apply exclusivity cuts, which basically means requiring both forward protons to be tagged in FPDs and requiring large rapidity gaps. They are very powerful in reducing the inclusive backgrounds, but the price to pay is a rather low signal cross section. In the presence of pileup, rapidity gaps are filled by soft particles, so we can require all final state objects only to be well isolated from each other and not to be accompanied by large numbers of particles. By having protons tagged on both sides of the FPD, we can also utilize time-of-flight (TOF) detectors, which are very useful for suppressing a combinatorial background coming from pileup; see, e.g., Refs. [38-40].

The signal processes (the $t \bar{t}$ production in $\gamma \gamma, \gamma \mathbb{P}$, and $\mathbb{P P}$ processes) and the exclusive $W W$ production, $\gamma \gamma \rightarrow W W$, for the background are analyzed using FPMC [16], while the background from photoproduction of the single top, $\gamma \mathbb{P} \rightarrow W t$, is studied using MadGraph5 [41] and PYTHIA8 [42] (for previous studies in inclusive processes see, e.g., Refs. [43-45]). The main background, namely, the inclusive $t \bar{t}$ production with pileup, is generated using MadGraph5 and PYTHIA8. While the signal processes and the inclusive background are studied at detector level, the contamination by the exclusive backgrounds is estimated at generator level. Detector effects and pileup mixing are incorporated using DELPHES [46] with an input card with ATLAS detector specifications. For both the exclusive and inclusive $t \bar{t}$ processes, the mass of the top quark is set to the value of $174.0 \mathrm{GeV}$. For FPDs we assume fully efficient reconstruction in the range $0.015<\xi_{1,2}<0.15$, where $\xi_{1,2}=1-p_{z 1,2} / E_{\text {beam }}$ is the fractional proton momentum loss on either side of the interaction point (side 1 or 2 ). This, in principle, allows one to measure masses of the central system by the missing mass method, $M=\sqrt{\xi_{1} \xi_{2} s}$, starting from about $200 \mathrm{GeV}$. Large samples of the aforementioned processes have been generated corresponding to luminosities that sufficiently exceed those delivered or expected to be delivered by the LHC in the future. The cuts used for these generations are looser than those used in the analysis to account for detector effects (e.g., $0.005<\xi_{1,2}<0.20$ ).

\section{A. Backgrounds}

Considering the signal to be a sum of contributions from all three signal processes $(t \bar{t}$ produced in $\gamma \gamma, \gamma \mathbb{P}$, and $\mathbb{P P}$ processes), we can divide relevant backgrounds to irreducible (where there are two intact protons on both sides) and reducible (where the hard-scale process itself does not provide intact protons but forms a dangerous background when overlaid with pileup interactions). We study in detail two relevant irreducible backgrounds, namely, the photoproduction of the single top quark in diffractive interactions, $\gamma \mathbb{P} \rightarrow W t$, and the exclusive $W W$ production, $\gamma \gamma \rightarrow W W$. Because of their relatively low cross sections, it is sufficient to study them at generator level. The cross section of the inclusive production of the $t \bar{t}$ pair is much higher, and therefore it is studied at detector level including pileup.

\section{B. Jets}

Jets are reconstructed from particles at generator level or from tracks at detector level using the anti- $k_{t}$ algorithm with a radius $R=0.4$, incorporated inside the FastJet package [47]. We require at least four jets in total, out of which at least two must be $b$-tagged, all having transverse momenta $E_{T \text {,jet }}>25 \mathrm{GeV}$ and pseudorapidity $\left|\eta_{\text {jet }}\right|<2.5$. At generator level, a jet is considered to be $b$-tagged if a $B$ hadron is found inside a cone of $R=0.4$ from the jet axis. The $b$-tagging at detector level is based on finding a parton inside a cone of $R=0.4$ from the jet axis. If the parton is a $b$-quark, the $b$-tag efficiency formula is applied to get a probability to find a $b$-tagged jet. If the parton is a light quark (or gluon), the misidentification rate for light-quark (or gluon) jets is applied to get a probability to misidentify a light-quark (or gluon) jet as the b-quark jet. All the b-tag efficiency and misidentification rates are given as functions of jet $E_{\mathrm{T}}$ and $\eta$. The efficiency formulas are provided inside the DELPHES input card and requiring at least two jets to be $b$-tagged means that at least two jets have the $b$-tagging efficiency greater than $70 \%$.

\section{Leptons}

In the analysis, we require at least one isolated lepton to be found, either an electron or a muon, possibly coming also from $\tau$ decays, with $E_{1}>25 \mathrm{GeV}$ and $\left|\eta_{1}\right|<2.5$. The lepton is considered to be isolated if the radius difference between the jet axis and the lepton is $\Delta R_{1, \mathrm{j}}>0.2$. The electron and muon reconstruction 
efficiencies specified in the DELPHES input card are applied as functions of $E_{\mathrm{T}}$ and $\eta$ at both generator and detector levels. The leptons are represented by corresponding particles at generator level and corresponding objects at detector level (electrons/positrons as clusters in calorimeters and muons as combined objects at muon spectrometers).

\section{Exclusivity cuts}

The exclusive or semiexclusive nature of our signal enables us to use two powerful cuts: we require (i) both intact protons to be detected by FPDs (the so-called double tag) and (ii) large rapidity gaps. Applying the first cut means accounting for the FPD acceptance, which is in general a function of $\xi$ and $p_{\mathrm{T}}$ of the intact proton. For simplicity and not losing much of generality, we assume that the acceptance is close to $100 \%$ in the range of $0.015<\xi_{1,2}<0.15$, where the $\xi_{1,2}$ values are obtained from protons at generator level. The inclusive backgrounds would naturally not survive such a cut, but the combinatorial background from, e.g., on average 50 pileup interactions in one event gives a non-negligible probability to see double-tagged events. Most often they come from two soft single-diffractive (SD) events each providing a proton in the FPD acceptance on one side from the interaction point (opposite to each other). Overlaid with a third pileup event, with a scale hard enough to pass thresholds of L1 triggers in a LHC experiment and realizing that each soft SD event has a rather large cross section, such a combination of three events can mimic our signal.

As we indicated above, due to the nonzero pileup studied in this analysis, it would be inefficient to require large regions of the central detector to be empty. Instead, we require all four jets and one lepton to be well separated from each other and not to be accompanied by large amounts of particles, so we introduce a cut based on the number of particles (or tracks if we work at detector level) from a narrow region around the primary vertex, the so-called $z$-vertex veto; see, e.g., Ref. [48]. This way the inclusive backgrounds are believed to be suppressed even in the presence of pileup. For this cut we count tracks with $p_{T}>$ $0.2 \mathrm{GeV}$ and $|\eta|<2.5$ (whose efficiencies and resolutions are properly taken into account by providing ATLAS specifications in the DELPHES card) if they are closer than $1 \mathrm{~mm}$ to the primary vertex in the $z$ coordinate. We count the total number of such tracks per event, which are at the same time distant from the four jets and one lepton, by requiring $\Delta R_{\text {trk }, \mathrm{j}}>0.4$ and $\Delta R_{\text {trk }, 1}>0.2$. We also studied two more scenarios, namely, $\left\{0.4<\Delta R_{\text {trk,j }}<0.8\right.$ and $\left.0.2<\Delta R_{\text {trk, },}<0.8\right\}$ and $\left\{0.4<\Delta R_{\text {trk,j }}<1.0\right.$ and $0.2<$ $\left.\Delta R_{\mathrm{trk}, 1}<1.0\right\}$ and found them to be less efficient than the first one. All these baseline cuts discussed above can then be grouped as follows:

(a) In total at least four nonoverlapping jets with $E_{T, \text { jet }}>$ $25 \mathrm{GeV}$ and $\left|\eta_{\text {jet }}\right|<2.5$.
TABLE I. Cuts used in this analysis.

\begin{tabular}{l}
\hline \multicolumn{1}{c}{ Cut } \\
\hline$N_{\text {jet }} \geq 4\left(E_{T}>25 \mathrm{GeV},|\eta|<2.5\right)$ \\
$N_{e / \mu} \geq 1\left(E_{T}>25 \mathrm{GeV},|\eta|<2.5\right)$ \\
$\Delta R(\mathrm{e} / \mu$, jet $)>0.2$ \\
$N_{b \text {-jet }} \geq 2$ \\
$0.015<\xi_{1,2}<0.15$ \\
$N_{\text {trk }}\left(p_{\mathrm{T}}>0.2 \mathrm{GeV},|\eta|<2.5,|\Delta z|<1 \mathrm{~mm}\right) \leq X$ \\
\hline \hline
\end{tabular}

(b) At least one electron or muon ( $\tau$ decays included) with $E_{\mathrm{T}, \mathrm{l}}>25 \mathrm{GeV}$ and $\left|\eta_{1}\right|<2.5$ isolated from all four jets, $\Delta R_{1, \mathrm{j}}>0.2$.

(c) At least two $b$-tagged jets. A jet is $b$-tagged if a $B$ hadron (generator level) or a $b$-quark (detector level) is found inside the jet.

(d) FPD acceptance $0.015<\xi_{1,2}<0.15$.

(e) Number of tracks with $p_{T \text {,trk }}>0.2 \mathrm{GeV}$ and $\left|\eta_{\text {trk }}\right|<$ 2.5 in the distance $\left|z_{\text {trk }}-z_{\mathrm{vtx}}\right|<1 \mathrm{~mm}$ from the primary vertex and $\Delta R_{\text {trk,j }}>0.4$ from the four jets and $\Delta R_{\text {trk, },}>0.2$ from one lepton must be smaller than a given value $X$.

They are summarized in Table I.

\section{RESULTS}

\section{A. Zero pileup scenario}

Owing to much lower cross sections of the two irreducible background processes studied in this analysis, both are considered only at zero pileup where the inclusive background is negligible.

The production cross section of the QED exclusive background with a $W W$ pair in the final state at $\sqrt{s}=$ $13 \mathrm{TeV}$ is $75.6 \mathrm{fb}$, which reduces to $34 \mathrm{fb}$ when taking only cases where both $W$ bosons decay hadronically, and further to $4.7 \mathrm{fb}$ when applying the jet $E_{T}$ and $\eta$ cuts. Understandably, the most suppressing cut is the requirement of at least one lepton with relatively high $E_{T}$ and which is well isolated from all four jets. The suppression factor of about 80 comes from the fact that leptons can originate only from semileptonic decays of heavy mesons (e.g., $D^{+} \rightarrow \mu^{+} \nu \pi^{0}$ ) and of kaons and pions inside jets and that this occurrence naturally drops with increasing lepton $E_{T}$. The lepton isolation criterion brings an additional suppression by a factor of 20 and, by requiring at least two $b$-tagged jets, we suppress the contribution of this background to a negligible level (see Table II).

The photoproduction cross section of the single top quark in diffractive interactions, $\gamma \mathbb{P} \rightarrow W t$, is $12 \mathrm{fb}$, as obtained from MadGraph5, and drops by a factor of 30 if the cuts on four jets and one lepton are applied. Another factor of 4 comes from requiring both intact protons to be found in the FPD acceptance. An effective cross section is of the order of $0.1 \mathrm{fb}$, which is about 30 times smaller than the 
TABLE II. Cut flow for the exclusive signal processes and inclusive background with zero pileup. The values marked as $\sim 0$ correspond to numbers which are sufficiently below $10^{-4}$.

\begin{tabular}{lcccccr}
\hline \hline Process & $\gamma \gamma$ & $\gamma \mathbb{P}$ & $\mathbb{P P}$ & Incl. $t \bar{t}+\mathrm{PU}$ & $\gamma \gamma \rightarrow W W$ & $\gamma \mathbb{P} \rightarrow W t$ \\
\hline Generated cross section $(\mathrm{fb})$ & 0.34 & 52.0 & 28.4 & 390000 & 75.6 & 12.0 \\
$N_{e / \mu} \geq 1\left(E_{T}>25 \mathrm{GeV},|\eta|<2.5\right)$ & 0.09 & 14.1 & 7.4 & 89991 & 0.06 & 2.0 \\
$N_{\text {jet }} \geq 4\left(E_{T}>25 \mathrm{GeV},|\eta|<2.5\right)$ & 0.02 & 3.9 & 2.0 & 36412 & 4.7 & 0.4 \\
$\Delta R(\mathrm{e} / \mu$, jet $)>0.2$ & 0.02 & 3.9 & 2.0 & 36412 & 0.003 & 0.4 \\
$N_{b \text {-jet }} \geq 2$ & 0.02 & 3.9 & 2.0 & 36412 & $10^{-4}$ & 0.4 \\
$0.015<\xi_{1,2}<0.15$ & 0.014 & 2.3 & 0.74 & $\sim 0$ & $\sim 0$ & 0.1 \\
\hline \hline
\end{tabular}

effective signal cross section and hence is considered to be negligible (see Table II).

A cut flow table for the zero pileup scenario is shown in Table II. In Figs. 2-4 we show control plots of some of the variables used in the event selection. All are obtained after applying cuts in Table I, except for the $N_{\text {trk }}$ cut. The distributions for the $\gamma \gamma, \gamma \mathbb{P}$, and $\mathbb{P} \mathbb{P}$ processes are obtained from FPMC, and the inclusive $t \bar{t}$ process from MadGraph $5+$ PYTHIA8. All distributions except for the missing mass in Fig. 2 are at detector level.

In Figs. 2 and 3 for signal processes, no pileup events are added. For illustration of the combinatorial background coming from pileup protons, we also show the inclusive $t \bar{t}$ background with, on average, ten pileup events per interaction. The size of this combinatorial background depends on the cross section of the hard-scale background and the amount of pileup (the inclusive $t \bar{t}$ process and $\langle\mu\rangle=10$ in this case). In Fig. 2, where we plot the missing mass obtained from the $\xi$ information at generator level, we see

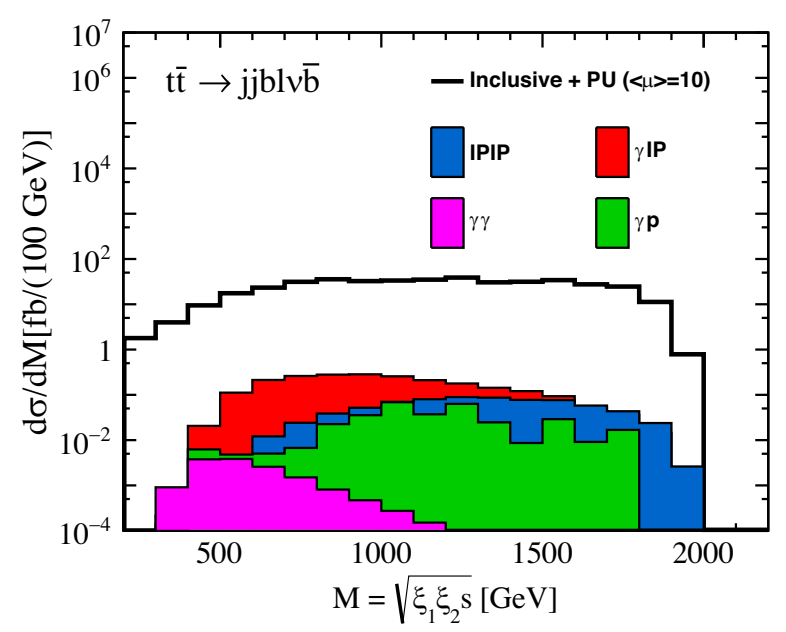

FIG. 2. Distribution of missing mass calculated using protons detected in FPDs at generator level after applying cuts in Table I, except for the $N_{\text {trk }}$ cut. Predictions for three (semi) exclusive signal processes are obtained with FPMC, predictions for the $\gamma \mathbb{P}$ background by MadGraph5, all without pileup, while the inclusive $t \bar{t}$ background was generated with MadGraph5 + PYTHIA8 and overlaid with pileup with $\langle\mu\rangle=10$ interactions per event. that the most prominent background has a shape of continuum. In Fig. 3 we show distributions of the mass (left panel) and pseudorapidity (right panel) of the $t \bar{t}$ pair. At this stage of analysis, we can already conclude that the yield of the exclusive $\gamma \gamma$ process is negligible. The irreducible backgrounds can be tamed to $3 \%$ of the signal or lower if more and better tailored cuts are applied.

Running at a very low or zero amount of pileup interactions per bunch crossing, for example, at $\mu \lesssim 1$, has a clear advantage in that the combinatorial background overlaid with a hard-scale inclusive background processes with large cross sections would become negligible. However, conceivable values of integrated luminosity (they would be smaller than $1 \mathrm{fb}^{-1}$ ) do not allow one to imagine measuring the top quark mass reliably since it would be based on fewer than ten events in the whole reachable range between $2 \cdot m_{t}$ and roughly $2.5 \mathrm{TeV}$. Relaxing the $p_{T}$ cuts for leptons (down to $5 \mathrm{GeV}$ ) and jets (down to $20 \mathrm{GeV}$ ) does not help since it would increase the signal statistics by only about $25 \%$.

\section{B. Nonzero pileup scenario}

In Figs. 2 and 3 we observe that the contamination by the mix of inclusive and combinatorial backgrounds is enormous and needs to be suppressed by special means. As discussed above, these are referred to as the exclusivity requirement and the suppression from using the TOF detector and are discussed in this section.

The hard-scale process for the inclusive $t \bar{t}$ production is generated using MadGraph5 [41], while showering and hadronization is done by PYTHIA8 [42]. The DELPHES package is then used for two purposes: (i) pileup mixing and (ii) inclusion of detector effects. First, it overlays the inclusive (hard-scale) $t \bar{t}$ events with pileup (usually of soft nature); in other words, it mixes one hard-scale event with a given number of pileup events such that the resulting distribution of the number of pileup events when integrated over all hard-scale events follows a Poissonian distributions with a mean equal to $\langle\mu\rangle$. We study in detail three working points, namely, $\langle\mu\rangle$ of 5, 10, and 50. The pileup events are generated by PYTHIA8 as minimum-bias events at $13 \mathrm{TeV}$ with default settings, i.e., multiparton interactions, initial as well as final state radiations are all switched on. Based on 

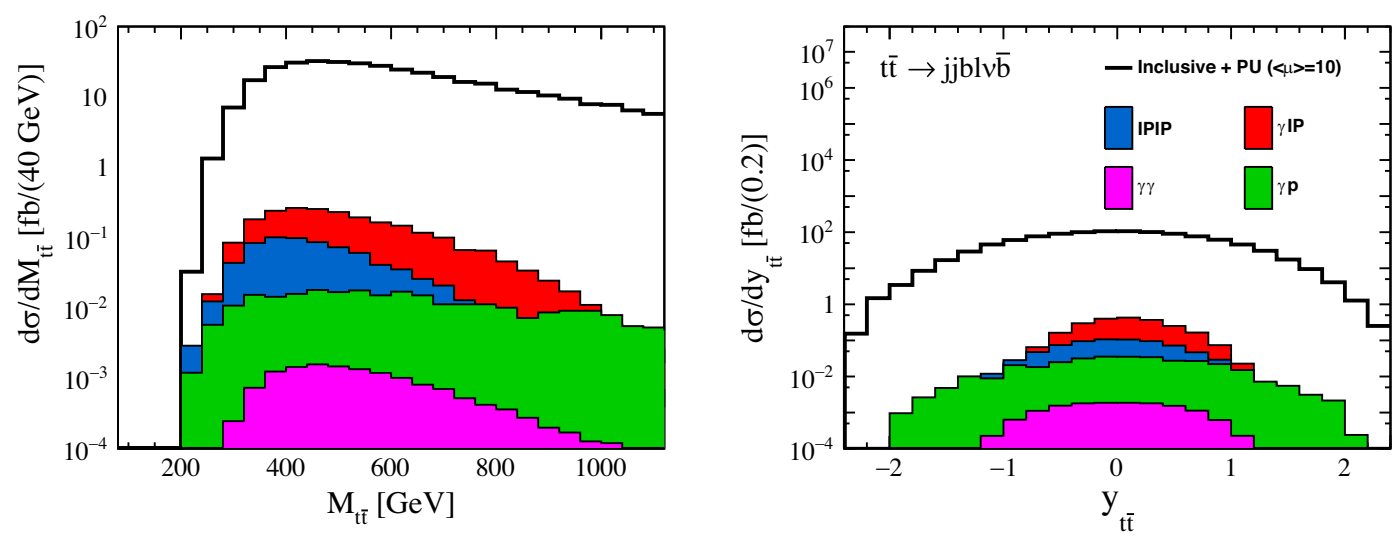

FIG. 3. Distribution of the mass (left panel) and pseudorapidity (right panel) of the $t \bar{t}$ pair at detector level and after applying cuts in Table I, except for the $N_{\text {trk }}$ cut. Predictions for three (semi)exclusive signal processes are obtained with FPMC, predictions for the $\gamma \mathbb{P}$ background by MadGraph5, all without pileup, while the inclusive $t \bar{t}$ background was generated with MadGraph5+PYTHIA8 and overlaid with pileup with $\langle\mu\rangle=10$ interactions per event.
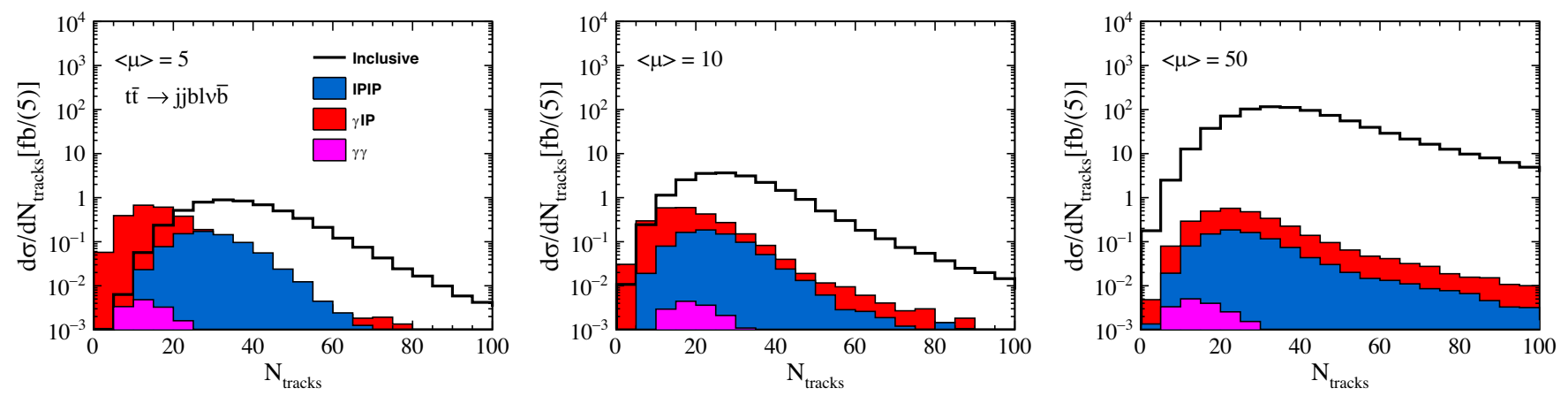

FIG. 4. Distribution of the number of tracks with $p_{T}>0.2 \mathrm{GeV}$ and $|\eta|<2.5$ outside all four jets and one lepton for three amounts of pileup events per interaction, $\langle\mu\rangle$, of 5,10, and 50, all at detector level and after applying cuts in Table I, except for the $N_{\text {trk }}$ cut. Predictions for three (semi)exclusive signal processes are obtained with FPMC, while the inclusive $t \bar{t}$ background was generated with MadGraph5+PYTHIA8.

the knowledge of the probability of seeing an intact proton from one minimum bias event in the FPD $\xi$ acceptance on one side, $P_{\mathrm{ST}}$, we are able to estimate the rate of fake double-tagged events and hence the combinatorial background from pileup. For the minimum-bias events generated as specified above, we get $P_{\mathrm{ST}}=1.4 \%$. The combinatorial factors representing the rates of fake double-tagged events for the studied values of $\langle\mu\rangle$ of 5, 10, and 50 are $0.0031,0.014$, and 0.246 , respectively (see Refs. [38-40] for more details and the $\langle\mu\rangle$ dependence of this background).

Second, DELPHES provides a fast simulation of all relevant detector features for which parameters are put in the input card. We used those specific for the ATLAS detector.

The distribution of number of tracks outside the four jets and one lepton for three amounts of pileup, namely, for $\langle\mu\rangle=5,10$, and 50, is shown in Fig. 4. Tracks are required to have $p_{T}>0.2 \mathrm{GeV}$ and be in the central tracker acceptance, $|\eta|<2.5$. When plotting these distributions, the effective cross sections of the mix of inclusive and pileup events are already scaled by the corresponding rates of fake double-tagged events (specified above) and by TOF suppression factors. The TOF suppression factors are 18.3, 17.3, and 10.8 for $\langle\mu\rangle=5,10$, and 50, respectively, under the assumption that the time resolution of TOF is $\sigma_{t}=10 \mathrm{ps}$ and the signal is collected in a $2 \sigma_{t}$ window. The effective cross sections after applying individual cuts from Table I and scaling by the rates of fake double-tagged events and by the TOF suppression are summarized in Table III. To suppress the dominant background further, we also added a cut on the missing mass evaluated by FPD and the mass of the top pair measured in the central detector.

To estimate the statistical significance, $\sigma$, and signal to background (S/B) ratio we consider three luminosity scenarios in terms of $\langle\mu\rangle$ and $\mathcal{L}$, where $\langle\mu\rangle$ represents the average number of pileup interactions per event (or the instantaneous luminosity) and $\mathcal{L}$ is the integrated luminosity. We assume $\mathcal{L}$ to be 10,30 , and $300 \mathrm{fb}^{-1}$ for $\langle\mu\rangle=5$, 10 , and 50 , respectively, and provide the information on the 
TABLE III. Cut flow for the effective cross sections in femtobarns for the exclusive signal processes and inclusive background with pileup overlaid with $\langle\mu\rangle=5,10$, and 50. The effect of the $\xi$ cut for the inclusive background with pileup is evaluated as a combinatorial background coming from the rate of fake double-tagged events. Suppression of pileup effects from using TOF information is based on $[38,39]$.

\begin{tabular}{lccc}
\hline \hline Process & $\gamma \mathbb{P}(\langle\mu\rangle=5 / 10 / 50)$ & $\mathbb{P P}(\langle\mu\rangle=5 / 10 / 50)$ & Incl. $\bar{t}+\operatorname{PU}(\langle\mu\rangle=5 / 10 / 50)$ \\
\hline Generated cross section (fb) & 52.0 & 28.4 & 390000 \\
$N_{e / \mu} \geq 1\left(E_{T}>25 \mathrm{GeV},|\eta|<2.5\right)$ & $14.1 / 14.2 / 13.4$ & $7.4 / 7.3 / 6.7$ & $90057 / 90042 / 82994$ \\
$N_{\text {jet }} \geq 4\left(E_{T}>25 \mathrm{GeV},|\eta|<2.5\right)$ & $4.2 / 4.4 / 5.4$ & $2.1 / 2.2 / 2.6$ & $38157 / 38928 / 42821$ \\
$\Delta R(\mathrm{e} / \mu$, jet $)>0.2$ & $4.2 / 4.4 / 5.4$ & $2.1 / 2.2 / 2.6$ & $38157 / 38928 / 42821$ \\
$N_{b \text {-jet }} \geq 2$ & $4.2 / 4.4 / 5.4$ & $2.1 / 2.2 / 2.6$ & $38157 / 38928 / 42821$ \\
$0.015<\xi_{1,2}<0.15$ & $2.4 / 2.6 / 3.2$ & $0.8 / 0.8 / 1.0$ & $118.2 / 423.3 / 10534$ \\
$m_{t \bar{t}}<1000 \mathrm{GeV}, m_{X}>400 \mathrm{GeV}$ & $2.4 / 2.6 / 3.1$ & $0.8 / 0.8 / 1.0$ & $97.6 / 349.6 / 9107$ \\
TOF suppression & $2.4 / 2.6 / 2.4$ & $0.8 / 0.8 / 0.8$ & $5.3 / 20.2 / 843.2$ \\
$N_{\text {trk }} \leq 10$ & $0.45 / 0.44 / 0.14$ & $0.002 / 0.02 / 0.02$ & $0.006 / 0.35 / 2.7$ \\
$N_{\text {trk }} \leq 15$ & $1.12 / 1.12 / 0.60$ & $0.10 / 0.10 / 0.10$ & $0.12 / 1.39 / 15.4$ \\
$N_{\text {trk }} \leq 20$ & $1.73 / 1.76 / 1.20$ & $0.11 / 0.26 / 0.25$ & $0.29 / 3.94 / 52.8$ \\
$N_{\text {trk }} \leq 25$ & $2.11 / 2.16 / 1.80$ & $0.30 / 0.45 / 0.44$ & $0.81 / 7.49 / 123.9$ \\
\hline \hline
\end{tabular}

above for four $N_{\text {trk }}$ values in Table IV. The best combination of $\sigma$ and S/B parameters give track cuts $N_{\text {trk }} \leq 15$ or $N_{\text {trk }} \leq 20$ for all luminosity scenarios with significances of about 11,6 , and 3 going from the lowest to the largest luminosity scenario. These significances are rather insensitive to non-negligible uncertainties connected with the chosen value of the $S^{2}$ factor. In most cases, differences are below $5 \%$ and safely below $10 \%$ in the rest when changing the $S^{2}$ value from $3 \%$ to $2 \%$ or $4 \%$.

For a sensible measurement of the top quark mass, one would need a sufficient amount of signal events and a very low level of background contamination. As an example, we took the missing mass spectrum generated with a top quark mass of $171.7 \mathrm{GeV}$ (about $4 \sigma$ from the current top mass world average $172.8 \mathrm{GeV}$ ) and calculated the value of $\chi^{2}$, assuming that the template is modeled by a top quark mass of $172.8 \mathrm{GeV}$. For one of the best configurations of those studied in this analysis, namely, for $(\langle\mu\rangle, \mathcal{L})=(5,10)$, the differences are significantly below $1 \sigma$ (see the corresponding missing mass spectrum in Fig. 5 with signal and inclusive background after applying the $N_{\text {trk }} \leq 20$ cut).

When we enlarge the statistics 150 times, the $p$ values are $0.78,0.24$, and 0.42 for $N_{\text {trk }} \leq 15,20$, and 25,

TABLE IV. Summary of event yields for four values of $N_{\text {trk }}$ cut and for three luminosity scenarios $(\langle\mu\rangle, \mathcal{L})$ where $\mathcal{L}$ stands for the integrated luminosity in $\mathrm{fb}^{-1}$. For each scenario, the ratio of signal to background events, $N_{\mathrm{S}} / N_{\mathrm{B}}$, and a statistical significance is given.

\begin{tabular}{lccc}
\hline \hline$\left(\langle\mu\rangle, \mathcal{L}\left[\mathrm{fb}^{-1}\right]\right)$ & $(5,10)$ & $(10,30)$ & $(50,300)$ \\
\hline$N_{\text {trk }} \leq 10$ & $4.52 / 0.06,18.5$ & $13.8 / 10.5,4.3$ & $48.3 / 810.0,1.7$ \\
$N_{\text {trk }} \leq 15$ & $12.2 / 1.2,11.1$ & $36.6 / 41.7,5.7$ & $195 / 4616,2.9$ \\
$N_{\text {trk }} \leq 20$ & $18.3 / 2.9,10.7$ & $60.6 / 118.2,5.6$ & $429 / 15827,3.4$ \\
$N_{\text {trk }} \leq 25$ & $23.6 / 8.1,8.3$ & $78.3 / 224.7,5.2$ & $672 / 37195,3.5$ \\
\hline \hline
\end{tabular}

respectively. So only for the $N_{\text {trk }} \leq 20$ case we start to see a $1 \sigma$ effect. In other words, in order to distinguish a $1.2 \mathrm{GeV}$ difference in the measured top quark mass at a $1 \sigma$ significance (considering statistical uncertainties only), a sample of $1500 \mathrm{fb}^{-1}$ collected at $\langle\mu\rangle=5$ would be needed. In other studied luminosity and $N_{\text {trk }}$ points, the situation is even worse. Therefore we conclude that overall the situation with $\mathrm{S} / \mathrm{B}$ values does not give favorable prospects for measuring precisely the mass of the top quark. Let us note in this context that even lowering the TOF resolution to 5 ps would only lead to halving the combinatorial background, which is clearly not sufficient from the statistical point of view.

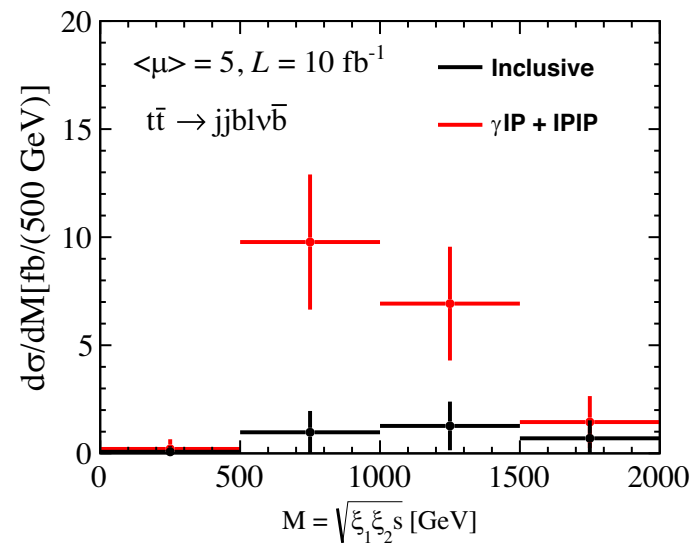

FIG. 5. Distribution of missing mass calculated using protons detected in FPDs at generator level after applying cuts in Table I and the corresponding TOF suppression and $N_{\text {trk }} \leq 20$ cut. Predictions for the two semiexclusive signal processes are obtained with FPMC, while the inclusive $t \bar{t}$ background was generated with MadGraph5 + PYTHIA8. All are overlaid with pileup with $\langle\mu\rangle=5$ interactions per event and numbers of events correspond to the integrated luminosity of $10 \mathrm{fb}^{-1}$. 


\section{SUMMARY}

We studied in detail prospects for measuring the $t \bar{t}$ pair produced in the exclusive $(\gamma \gamma)$ and semiexclusive $(\gamma \mathbb{P}$ and $\mathbb{P}(\mathbb{P})$ processes. We analyzed four luminosity scenarios, going from zero pileup up to $\langle\mu\rangle$ of 50 with corresponding assumed integral luminosities of up to $300 \mathrm{fb}^{-1}$. With the help of DELPHES, the main effects of detector acceptance and resolutions as well as the effect of pileup background were included in the analysis procedure. Good prospects for observing the exclusive signal over a mixture of inclusive and combinatorial background are achieved for all luminosity scenarios, although a good separation between the two is observed for rather low amounts of pileup, typically lower than $\langle\mu\rangle$ of 50 . Statistical significances evaluated from estimated numbers of signal and background events are around 3 for the highest luminosity scenario $\left(\langle\mu\rangle, \mathcal{L}\left[\mathrm{fb}^{-1}\right]\right)=(50,300)$, about 6 for the $(10,30)$ and 11 for the $(5,10)$ scenarios. From a simple statistical analysis, we find that these significances are still not sufficient for a determination of the top quark mass that would be competitive with inclusive methods. Much higher statistics would have to be developed with the current experimental procedure or more sophisticated procedures to suppress the dominant background.

\section{ACKNOWLEDGMENTS}

The authors thank James Howarth for the initial inspiration of this study. V. P. G. thanks the members of the Institute of Physics of the Czech Academy of Science for the warm hospitality at the beginning of this project. This work was partially financed by the Brazilian funding agencies CNPq, CAPES, FAPERGS, FAPERJ, and INCT-FNA (Grants No. 464898/2014-5 and No. 88887.461636/2019-00). M. T. is supported by MEYS of the Czech Republic by Project No. LTT17018.
[1] A. Andronic et al., Eur. Phys. J. C 76, 107 (2016).

[2] U. Husemann, Prog. Part. Nucl. Phys. 95, 48 (2017).

[3] S. Fayazbakhsh, S. T. Monfared, and M. Mohammadi Najafabadi, Phys. Rev. D 92, 014006 (2015).

[4] J. Howarth, arXiv:2008.04249.

[5] G. Aad et al. (ATLAS Collaboration), J. High Energy Phys. 11 (2019) 150.

[6] G. Aad et al. (ATLAS Collaboration), Eur. Phys. J. C 79, 1028 (2019).

[7] G. Aad et al. (ATLAS Collaboration), Eur. Phys. J. C 80, 528 (2020).

[8] A. M. Sirunyan et al. (CMS Collaboration), Phys. Rev. D 97, 112003 (2018).

[9] A. M. Sirunyan et al. (CMS Collaboration), J. High Energy Phys. 04 (2018) 060.

[10] A. M. Sirunyan et al. (CMS Collaboration), Eur. Phys. J. C 79, 368 (2019).

[11] A. Hebecker, Phys. Rep. 331, 1 (2000); L. Schoeffel, Prog. Part. Nucl. Phys. 65, 9 (2010); M. G. Albrow, T. D. Coughlin, and J. R. Forshaw, Prog. Part. Nucl. Phys. 65, 149 (2010).

[12] L. Adamczyk et al., CERN Reports No. CERN-LHCC2015-009 and No. ATLAS-TDR-024, 2015.

[13] M. Tasevsky (ATLAS Collaboration), AIP Conf. Proc. 1654, 090001 (2015).

[14] M. Albrow et al. (CMS and TOTEM Collaborations), CERN Reports No. CERN-LHCC-2014-021, No. TOTEM-TDR-003, and No. CMS-TDR-13, 2014.

[15] G. Ingelman and P. E. Schlein, Phys. Lett. 152B, 256 (1985).

[16] M. Boonekamp, A. Dechambre, V. Juranek, O. Kepka, M. Rangel, C. Royon, and R. Staszewski, arXiv:1102.2531.

[17] C. A. Bertulani and G. Baur, Phys. Rep. 163, 299 (1988); C. A. Bertulani, S. R. Klein, and J. Nystrand, Annu. Rev. Nucl. Part. Sci. 55, 271 (2005); A. J. Baltz et al., G. Baur,
D. Denterria, L. Frankfurt, F. Gelis, V. Guzey, K. Hencken, Y. Kharlov, M. Klasen, and S. Klein, Phys. Rep. 458, 1 (2008); K. Akiba et al. (LHC Forward Physics Working Group), J. Phys. G 43, 110201 (2016).

[18] V. M. Budnev, I. F. Ginzburg, G. V. Meledin, and V. G. Serbo, Phys. Rep. 15, 181 (1975).

[19] B. A. Kniehl, Phys. Lett. B 254, 267 (1991).

[20] M. Luszczak, L. Forthomme, W. Schafer, and A. Szczurek, J. High Energy Phys. 02 (2019) 100.

[21] V. P. Goncalves, Phys. Rev. D 88, 054025 (2013).

[22] A. Aktas et al. (H1 Collaboration), Eur. Phys. J. C 48, 715 (2006).

[23] V. P. Goncalves and M. M. Machado, Phys. Rev. D 85, 054019 (2012).

[24] M. Luszczak, R. Maciula, and A. Szczurek, Phys. Rev. D 91, 054024 (2015).

[25] V. P. Goncalves, C. Potterat, and M. S. Rangel, Phys. Rev. D 93, 034038 (2016).

[26] M. Goharipour, H. Khanpour, and V. Guzey, Eur. Phys. J. C 78, 309 (2018).

[27] J. D. Bjorken, Phys. Rev. D 47, 101 (1993).

[28] V. A. Khoze, A. D. Martin, and M. G. Ryskin, Int. J. Mod. Phys. A 30, 1542004 (2015).

[29] E. Gotsman, E. Levin, and U. Maor, Int. J. Mod. Phys. A 30, 1542005 (2015).

[30] C. O. Rasmussen and T. Sjöstrand, J. High Energy Phys. 02 (2016) 142.

[31] J. C. Collins, Phys. Rev. D 57, 3051 (1998); 61, 019902(E) (1999).

[32] M. G. Ryskin, A. D. Martin, V. A. Khoze, and A. G. Shuvaev, J. Phys. G 36, 093001 (2009).

[33] M. Luszczak, R. Maciula, and A. Szczurek, Phys. Rev. D 84, 114018 (2011). 
[34] V. P. Goncalves, M. M. Jaime, D. E. Martins, and M. S. Rangel, Phys. Rev. D 97, 074024 (2018).

[35] C. Brenner Mariotto, V. P. Goncalves, and R. Palota da Silva, Phys. Rev. D 98, 014028 (2018); V. P. Goncalves and R. Palota da Silva, Phys. Rev. D 101, 034025 (2020).

[36] V. A. Khoze, A. D. Martin, and M. G. Ryskin, Eur. Phys. J. C 18, 167 (2000).

[37] G. Aad et al. (ATLAS Collaboration), Eur. Phys. J. C 76, 538 (2016).

[38] L. A. Harland-Lang, V. A. Khoze, M. G. Ryskin, and M. Tasevsky, J. High Energy Phys. 04 (2019) 010.

[39] M. Tasevsky, Int. J. Mod. Phys. A 29, 1446012 (2014).

[40] K. Cerny, M. Tasevsky, T. Sykora, and R. Zlebcik, arXiv: 2010.00237.

[41] J. Alwall, M. Herquet, F. Maltoni, O. Mattelaer, and T. Stelzer, J. High Energy Phys. 06 (2011) 128.
[42] T. Sjöstrand, S. Ask, J. R. Christiansen, R. Corke, N. Desai, P. Ilten, S. Mrenna, S. Prestel, C. O. Rasmussen, and P.Z. Skands, Comput. Phys. Commun. 191, 159 (2015).

[43] J. de Favereau de Jeneret and S. Ovyn, Nucl. Phys. B, Proc. Suppl. 179-180, 277 (2008).

[44] H. Sun, Nucl. Phys. B886, 691 (2014).

[45] H. Sun, W. Liu, X. J. Wang, Y. J. Zhou, and H. S. Hou, J. High Energy Phys. 02 (2015) 064.

[46] J. de Favereau, C. Delaere, P. Demin, A. Giammanco, V. Lemaître, A. Mertens, and M. Selvaggi (DELPHES 3 Collaboration), J. High Energy Phys. 02 (2014) 057.

[47] M. Cacciari, G. P. Salam, and G. Soyez, Eur. Phys. J. C 72, 1896 (2012).

[48] M. Aaboud et al. (ATLAS Collaboration), Phys. Lett. B 777, 303 (2018). 